\title{
Photoluminescence quenching and photocatalytic enhancement of Pr-doped ZnO nanocrystals
}

\author{
N K DIVYA and P P PRADYUMNAN* \\ Department of Physics, University of Calicut, Malappuram 673635, India \\ *Author for correspondence (drpradyumnan@gmail.com) \\ MS received 10 March 2016; accepted 18 April 2017; published online 28 November 2017
}

\begin{abstract}
This work reports the synthesis of novel praseodymium (Pr)-doped ZnO nanocrystals with excellent photocatalytic activity through modified solid-state reaction route. The impacts of doping on the wurtzite hexagonal structure are analysed with X-ray diffraction (XRD) and Raman spectroscopy. The production of defect levels and the formation of Urbach energy within the system are confirmed using photoluminescence (PL) techniques and UV/Vis diffuse reflectance spectroscopy, respectively. The linear relationship between Urbach energy and band gap energy is elucidated from UV/Vis spectroscopy analysis. The changes happened to morphology by doping were tackled using scanning electron microscopy (SEM). The concentration quenching effect of PL emission with Pr doping is explained in detail. A three-fold enhancement in the photocatalytic activity was achieved with optimum Pr incorporation in $\mathrm{ZnO}$. This work successfully correlated PL quenching and enhanced photocatalytic activity with the defect production happened in $\mathrm{ZnO}$ system on $\mathrm{Pr}$ doping. The practical applicability of the photocatalyst was confirmed with a stability test.
\end{abstract}

Keywords. Solid-state reaction; X-ray diffraction; Raman spectroscopy; Urbach energy.

\section{Introduction}

Textile industries are the back bone of developing countries. The coloured waste water as well as the chemicals released from these industries play key role in the water pollution. Semiconductor photocatalysis is one of the successful methods to compete with these kind of pollutants. The oxides like $\mathrm{TiO}_{2}, \mathrm{SnO}_{2}, \mathrm{Cu}_{2} \mathrm{O}, \mathrm{ZnO}$, etc. are the commercially available photocatalysts [1-10]. The most preferred industrial catalyst, $\mathrm{TiO}_{2}$ possesses high optical stability and photocatalytic efficiency [11]. ZnO keeps a similar status as that of $\mathrm{TiO}_{2}$ and is an unavoidable candidate in the field of materials science. It is a n-type semiconductor oxide having a wide band gap $(3.37 \mathrm{eV})$ and large excitonic binding energy. It plays a significant role in the field of solar cells, luminescence, electrical, chemical and gas sensors, catalysis, electronics, optoelectronics, transducers, biomedical devices, etc. [12-15]. The low cost and nontoxic behaviour are the merits of $\mathrm{ZnO}$ compounds. Researchers have already succeeded in tuning the band gap of $\mathrm{ZnO}$ by adding impurities like transition metal ions [12]. Some of the researchers have focussed on rare-earth ion incorporation in $\mathrm{ZnO}$ to achieve high activity. There are several reports indicating the enhancement of photocatalytic activity of $\mathrm{ZnO}$ by the doping of $\mathrm{Co}, \mathrm{Sm}, \mathrm{Tb}, \mathrm{Ho}$ and $\mathrm{Er}$ [13-16]. The synthesis methods have a very important role in determining the properties of $\mathrm{ZnO}$ [14]. Most of the reported synthesis methods are solution methods with very less yield and low purity that limits the industrial preparation of the sample for various applications.

The solid-state reaction route has an advantage of very high yield. But the method involves high temperature processing of samples. Because of this high temperature heating process, the production of nanoparticle through this method is very rare. Here, we report the successful production of $\mathrm{ZnO}$ nanoparticles with enhanced photocatalytic activity at optimum Pr incorporation by modified solid-state reaction route. The as-prepared samples were characterized by X-ray diffraction (XRD), Raman spectroscopy, energy dispersive $\mathrm{X}$-ray spectroscopy (EDS), scanning electron microscopy (SEM), UV/Vis diffuse reflectance spectroscopy and photoluminescence (PL) techniques. This work correlates the defect production happened in $\mathrm{Pr}$-doped $\mathrm{ZnO}$ systems with photocatalytic activity and PL.

\section{Experimental}

\subsection{Materials and preparation}

The samples were prepared through modified solid-state reaction route. The method of modified solid-state reaction facilitates high yield production of samples with high 
purity. $\mathrm{ZnO}$ (Sigma Aldrich product, $99.99 \%$ purity) and $\mathrm{Pr}_{2} \mathrm{O}_{3}$ (Sigma Aldrich product, $99.99 \%$ purity) were mixed thoroughly in an agate mortar and made into slurry with $\mathrm{LiOH} \cdot \mathrm{H}_{2} \mathrm{O}$ (Merck product, $99.99 \%$ purity) and ethanol (Merck product, $99.99 \%$ purity). The slurry was then transferred into a Petri dish and dried at $100^{\circ} \mathrm{C}$ in an oven. Then, the obtained powder sample was well ground for $1 \mathrm{~h}$ using a mortar and pestle and made in to pellets using hydraulic pelletizer at a pressure of 8 Torr. The pellets were fired at $900^{\circ} \mathrm{C}$ using a muffle furnace. Then, the heat-treated pellets were again ground for $15 \mathrm{~min}$ and used as the sample for the studies. The prepared samples are $\mathrm{ZnO}$ and $\mathrm{Zn}_{(1-x)} \operatorname{Pr}_{x} \mathrm{O}\left(\mathrm{Zn}_{0.98} \operatorname{Pr}_{0.02} \mathrm{O}, \mathrm{Zn}_{0.95} \operatorname{Pr}_{0.05} \mathrm{O}\right.$ and $\left.\mathrm{Zn}_{0.92} \mathrm{Pr}_{0.08} \mathrm{O}\right)$.

\section{Results and discussion}

Rigaku Miniflex 600 machine with $\mathrm{CuK} \alpha$ radiation $(\lambda=$ $1.5406 \AA$ ) is used for checking phase purity and getting structural information. X-ray diffraction patterns of pure and doped- $\mathrm{ZnO}$ micro/nanocrystals are shown in figure 1a. All the prepared samples showed high crystallinity and kept the hexagonal wurtzite structure of pure $\mathrm{ZnO}$. The XRD patterns are well matched with the standard ICDD card no. 01-0749940. The incorporation of $\mathrm{Pr}$ in $\mathrm{ZnO}$ is confirmed from the shift observed in the peak corresponding to (101) plane and is shown in figure $1 \mathrm{~b}$. Peak is shifted towards the higher angle side with both the small and the excess incorporation of Pr, indicating the increase in lattice strain. At the optimum incorporation of $\mathrm{Pr}$, the peak is shifted towards the lower angle side indicating the minimum lattice strain.

The Scherrer formula,

$$
D=0.9 \lambda / \beta \cos \theta
$$

where $\lambda$ is the wavelength of $\mathrm{CuK} \alpha$ radiation (1.514 $\AA$ ), $\theta$ the peak position and $\beta$ the FWHM meant to calculate the crystallite size. The average crystallite size is found to be decreasing with an increase in doping concentration. Pure $\mathrm{ZnO}$ nanoparticles with average crystallite size of $67.71 \mathrm{~nm}$ is reduced to $56.21 \mathrm{~nm}$ with $0.08 \mathrm{wt} \%$ doping of praseodymium. The incorporation of larger $\mathrm{Pr}$ ions in the host $\mathrm{ZnO}$ lattice reduces the nucleation and subsequent growth rate of $\mathrm{ZnO}$ crystals. This may be the cause behind the reduction in crystallite size with doping. Similar results were reported in the literature [16-18].

Crystallization, structural disorder and defects at microand nano-levels can be studied with microRaman spectra analysis of the prepared samples [19]. Raman spectroscopic analysis was done with Dilor XY-800 Spectrometer that was equipped with a charge-coupled device camera (radiation excitation was done with $532 \mathrm{~nm}$ ). $\mathrm{ZnO}$ has wurtzite hexagonal structure with four atoms per unit cell leading to 12 phonon branches, nine optical and three acoustic. According to the theoretical calculations, there is one A1 branch, with both Raman and Infrared active, one doubly
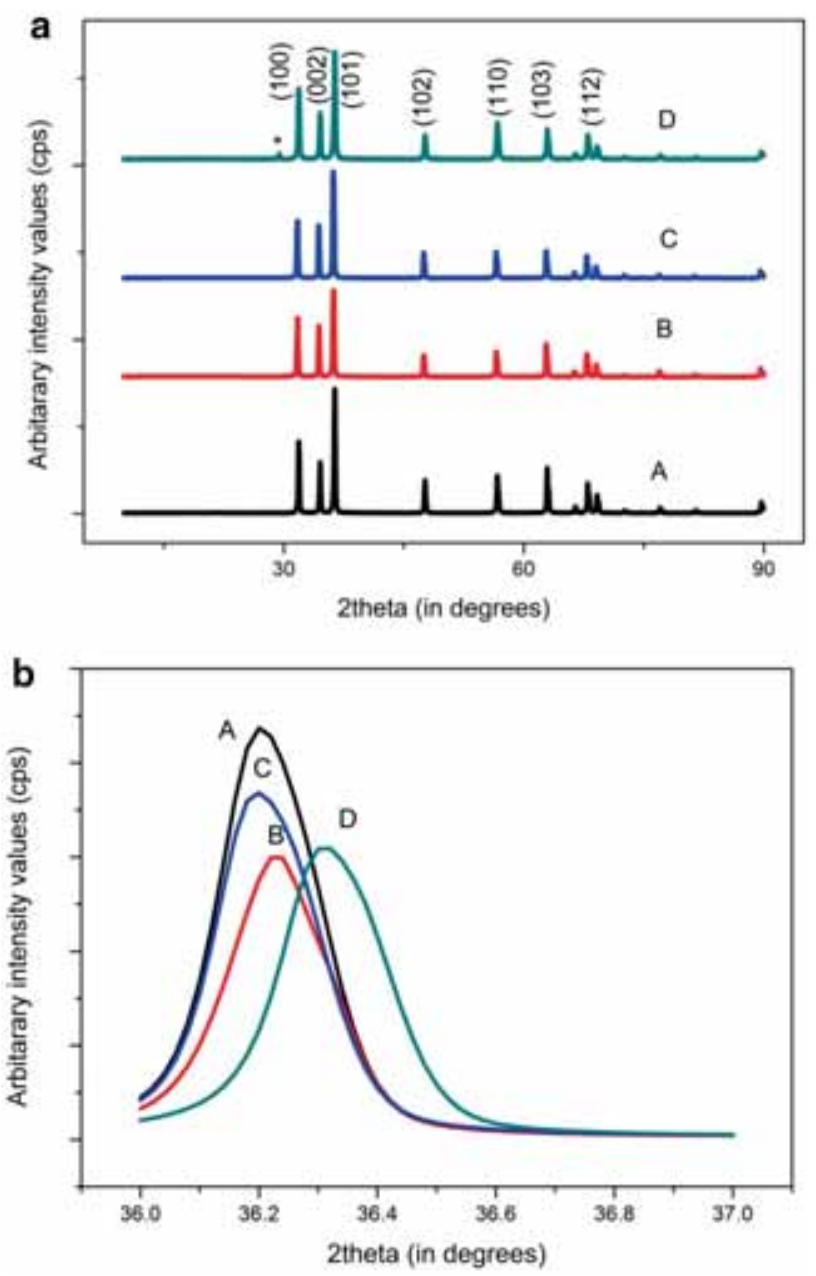

Figure 1. (a) XRD pattern of the samples. A, pure $\mathrm{ZnO}$; $\mathrm{B}$, 0.02 wt $\%$ Pr-doped $\mathrm{ZnO}$; C, 0.05 wt\% Pr-doped $\mathrm{ZnO}$; and $\mathrm{D}$, 0.08 wt $\%$ Pr-doped ZnO. (b) Shift observed for peak corresponding to (101) plane. A, Pure $\mathrm{ZnO} ; \mathrm{B}, 0.02 \mathrm{wt} \%$ Pr-doped $\mathrm{ZnO} ; \mathrm{C}$, 0.05 wt \% Pr-doped ZnO; and D, 0.08 wt $\%$ Pr-doped $\mathrm{ZnO}$.

degenerate E1 branch, two doubly degenerate E2 branches, Raman active only in two B branches, Raman inactive near the centre of the Brillion zone $[19,20]$. But A1 and E1 modes may split into transverse optical (TO) and longitudinal optical (LO) phonon branches. The obtained Raman spectra of pure $\mathrm{ZnO}$ and doped- $\mathrm{ZnO}$ samples are shown in figure 2 . For pure $\mathrm{ZnO}$, the spectrum shows usual peaks at $572 \mathrm{~cm}^{-1}$ corresponding to A1 LO phonon and a peak at $436.48 \mathrm{~cm}^{-1}$ corresponding to $\mathrm{E}_{2}$ high phonon. Mode at $436.48 \mathrm{~cm}^{-1}$ arises due to the vibration of oxygen sublattice. The doping of large Pr ions into the $\mathrm{ZnO}$ lattice will introduce lattice defects and disorder into the host. The oxygen vacancies will decrease the average atomic mass and may cause mass fluctuations at oxygen positions [19]. This results in the shifting of peak E2 (high) towards the low frequency side with Pr doping, observed in figure 2. The intensity of Raman peaks is found to be decreased with Pr doping. This can be explained with 


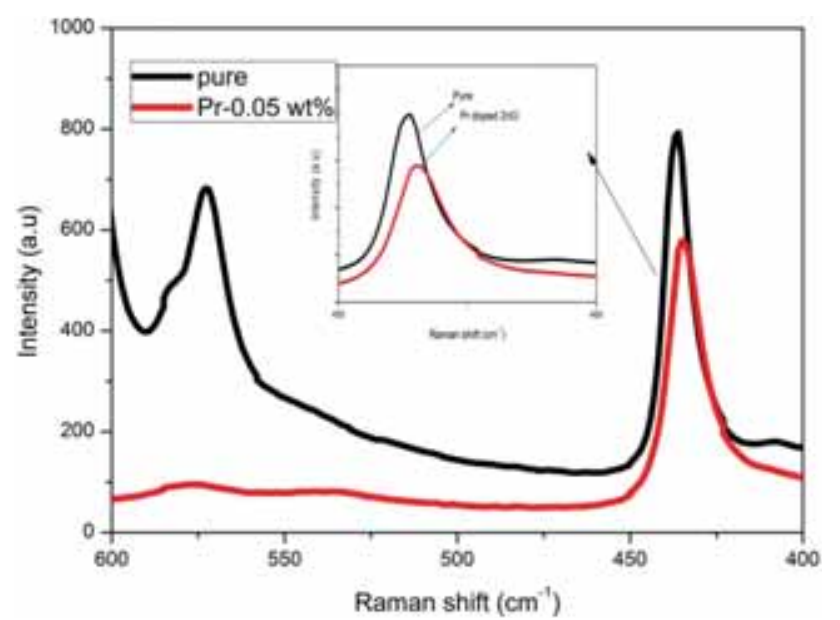

Figure 2. Room temperature microraman spectra of pure and $0.05 \mathrm{wt} \%$ Pr-doped samples ( $\mathrm{E}_{2}$ (high) peak is enlarged and shown in inset).

the size effect of Raman modes. The intensity of Raman peaks will decrease with a decrease in size of the particles [20]. This is in complete agreement with the XRD and SEM results.

Energy dispersive X-ray analysis (EDAX) equipment of Hitatchi is used to confirm the purity of the samples. The samples were cleaned by $\mathrm{Ar}^{+}$ion-beam sputtering to remove surface contamination if any. Figure $3 a$ and $b$ are the energy dispersion spectra of undoped $\mathrm{ZnO}$ and doped $\left(\mathrm{Pr}^{3+}\right.$ $0.05 \mathrm{wt} \%$ ) ceramics, respectively. From figure $3 \mathrm{a}$, the purity of parent sample is confirmed. There are no impurities in the parent $\mathrm{ZnO}$ sample. The incorporation of $\mathrm{Pr}^{3+}$ ions in the $\mathrm{ZnO}$ crystal lattice is confirmed from figure $3 b$.

SEM and Hitachi SU6600 variable pressure field emission were used for getting the morphology of the prepared samples. The incorporation of large praseodymium ions in $\mathrm{Zn}$ lattice sites makes changes in the morphology of crystallites. SEM images of undoped and Pr-doped $\mathrm{ZnO}$ are shown in figure $4 \mathrm{a}-\mathrm{d}$, where particles are in the micrometre and nanometre sizes. In figure $4 \mathrm{a}$, the distribution of size as well as shapes of pure $\mathrm{ZnO}$ particles are found to be nonuniform and this may be due to the damages occurred during the recrystallization of $\mathrm{ZnO}$ lattice at the high temperature heating process [16]. Pure $\mathrm{ZnO}$ particles are found to be irregular in shape and are found to be in the micrometre range as shown in figure $4 \mathrm{a}$. Pr-doped samples have relatively uniform distribution as well as small size. Figure $4 \mathrm{~b}-\mathrm{d}$ is corresponding to the doping of $\operatorname{Pr}$ in $0.02,0.05$ and $0.08 \mathrm{wt} \%$, respectively. The size of particles decreases with increases in Pr concentration and minimum size is observed for optimum concentration, $0.05 \mathrm{wt} \%$ Pr-doped samples. This is due to the effect of doping on the nucleation mechanism in the growth process. The ionic radius of $\mathrm{Pr}^{3+}$ ions is larger than that of $\mathrm{Zn}^{2+}$ ions, so a restrained nucleation rate and a subsequent lower growth rate of samples may occur, which results in the reduction of particle size. There is a chance to increase the particle size with excess addition of $\mathrm{Pr}$ in $\mathrm{ZnO}$. At low impurity addition, $\mathrm{Pr}^{3+}$ ions may homogeneously dissolve in the host matrix within the solubility limit and at higher concentration, the excess $\mathrm{Pr}^{3+}$ ions may be accumulated at the grain boundaries and produce large grains.

Jasco V-550 UV/Vis spectrophotometer is used for the optical band gap studies and degradation analysis. The fundamental optical band gap energy of the samples was computed by extrapolating the linear part of $(F(R) h v)^{2}$ to the $h v$ axis in the modified Kubelka-Munk function $v s . h v$ graph [21-23]. The variation of energy gap with praseodymium concentration in the sample is shown in figure 5. The band gap reduction happened on doping is evident from the combined graph, figure 5 . The decrease of band gap energy (red shift) values with the increase in Pr concentration is evident from the graph. The doping has introduced defect levels between the conduction band and valence band, thus resulted in the reduction of band gap energy. In other words, the impurities or defects caused the band tailing in the band gap energy of $\mathrm{ZnO}$. To analyse the effect of band tailing, band tailing energy (Urbach energy) [24] calculation can be done.

The absorption coefficient $(\alpha)$ of the prepared samples can be calculated with

$$
\alpha=A / t,
$$
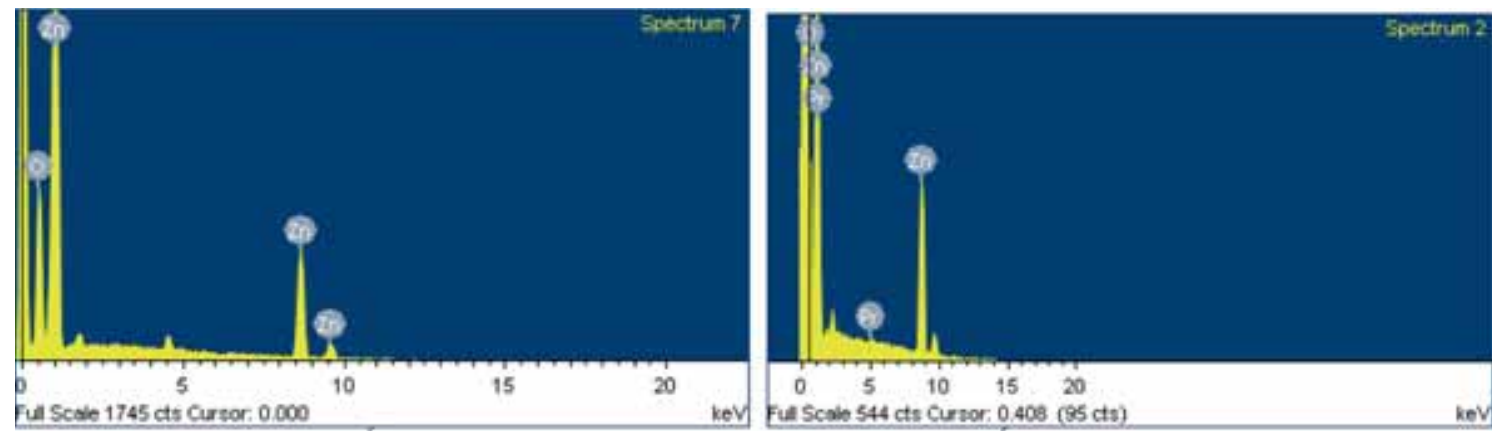

Figure 3. Energy dispersive spectra: (a) pure and (b) doped $\left(\mathrm{Pr}^{3+}-0.05 \mathrm{wt} \%\right)$ samples. 

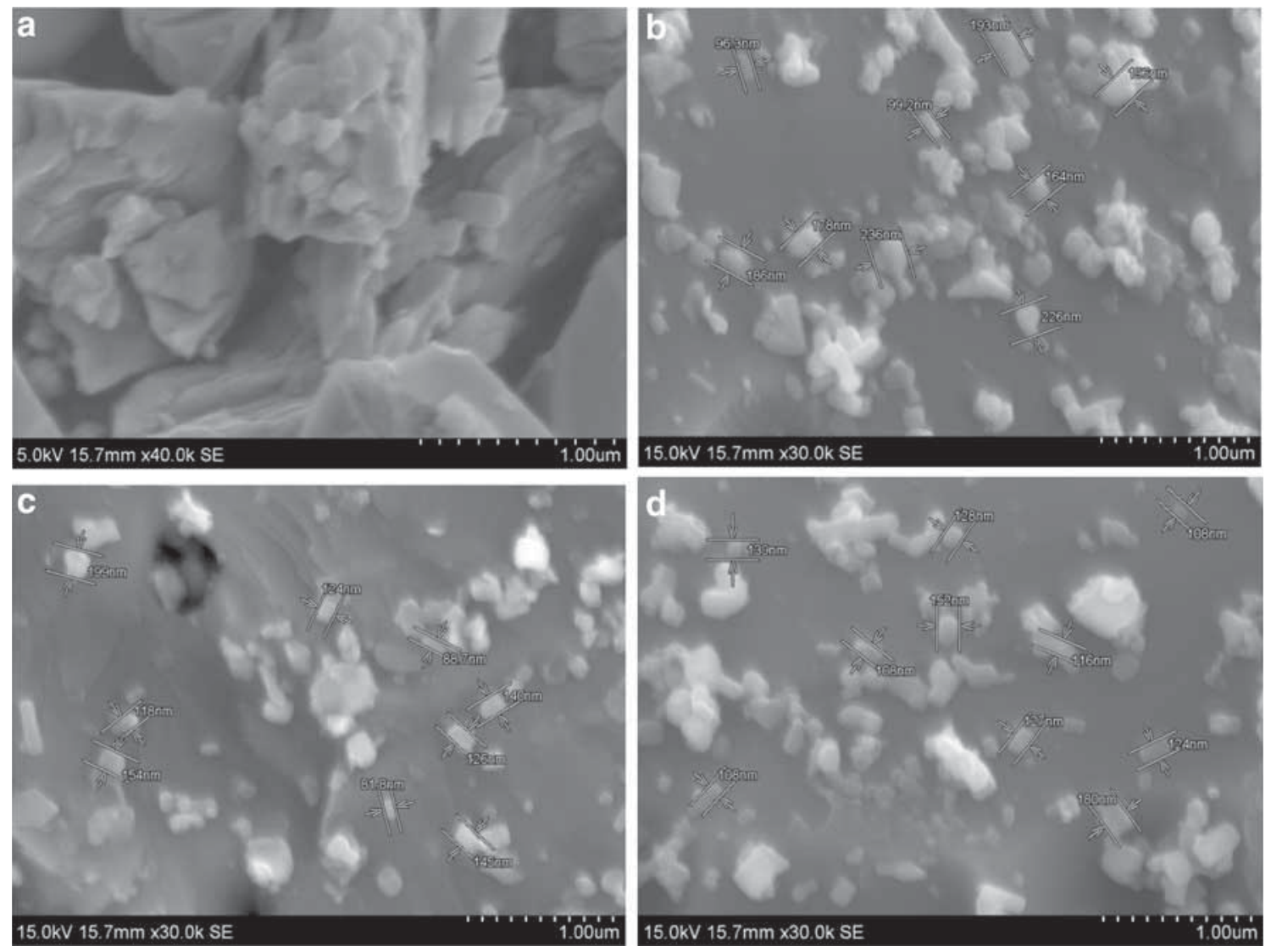

Figure 4. SEM images of samples: (a) pure $\mathrm{ZnO}$, (b) $0.02 \mathrm{wt} \% \mathrm{Pr}$-doped $\mathrm{ZnO}$, (c) $0.05 \mathrm{wt} \%$ Pr-doped $\mathrm{ZnO}$ and (d) 0.08 wt $\%$ Pr-doped $\mathrm{ZnO}$.

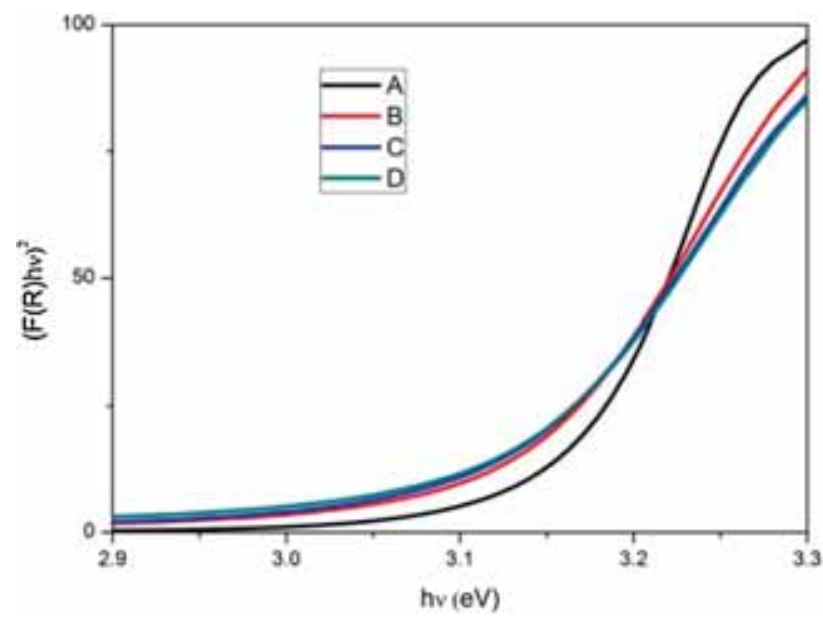

Figure 5. Modified Kubelka-Munk function $v s . h v$ graph of prepared samples. A, pure $\mathrm{ZnO}$; B, 0.02 wt\% Pr-doped $\mathrm{ZnO}$; C, 0.05 wt $\%$ Pr-doped $\mathrm{ZnO}$; and D, 0.08 wt $\%$ Pr-doped $\mathrm{ZnO}$.

where $A$ is the absorbance and $t$ the thickness of the sample holder. From the relation,

$$
\alpha=\alpha_{0} \exp \left(E / E_{\mathrm{u}}\right)
$$

where $\alpha_{0}$ is a constant, $E$ the photon energy and $E_{\mathrm{u}}$ the Urbach energy, $E_{\mathrm{u}}$ can be calculated as the inverse slope of $\ln (\alpha) v s$. $E$ graph. Increase in $E_{\mathrm{u}}$ or tailing energy actually reduces the band gap energy of the semiconductor. $E_{\mathrm{u}}$ of various prepared samples are calculated from $\ln (\alpha) v s$. $E$ graphs (supplementary information). The increase of $E_{\mathrm{u}}$ with the increase in Pr-doping concentration is shown in figure 6. This is again a confirmation of the band tailing effect happened in the $\mathrm{ZnO}$ matrix through doping. Band tailing affects the photocatalytic activity positively. As the band gap energy decreases, the probability of the transition of electrons within the bandgap with low energy photons is high. In other words, the increase of Urbach energy enhances the light absorption together with the available number of charge carriers within the system.

\section{PL study}

The photoluminescent studies were done with Horiba Jobin Yvon fluoromax 4 spectrofluorometer. Figure 7 shows the fluorescence spectra of undoped and Pr-doped $\mathrm{ZnO}$ powder excited at a wavelength of $325 \mathrm{~nm}$ at room temperature in the UV and visible regions, respectively. The intensity of PL emission was found to be decreased with increase in $\mathrm{Pr}$ 


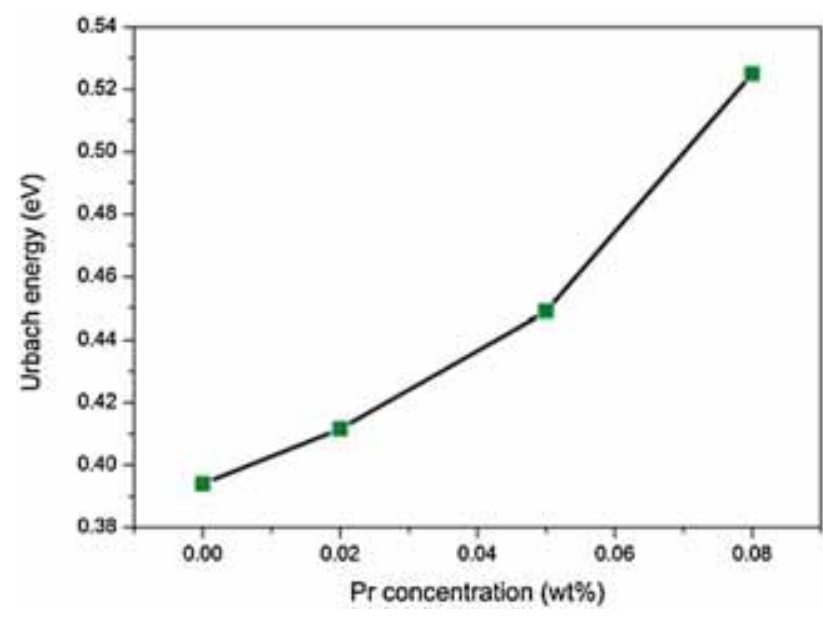

Figure 6. Variation of Urbach energy with Pr concentration.

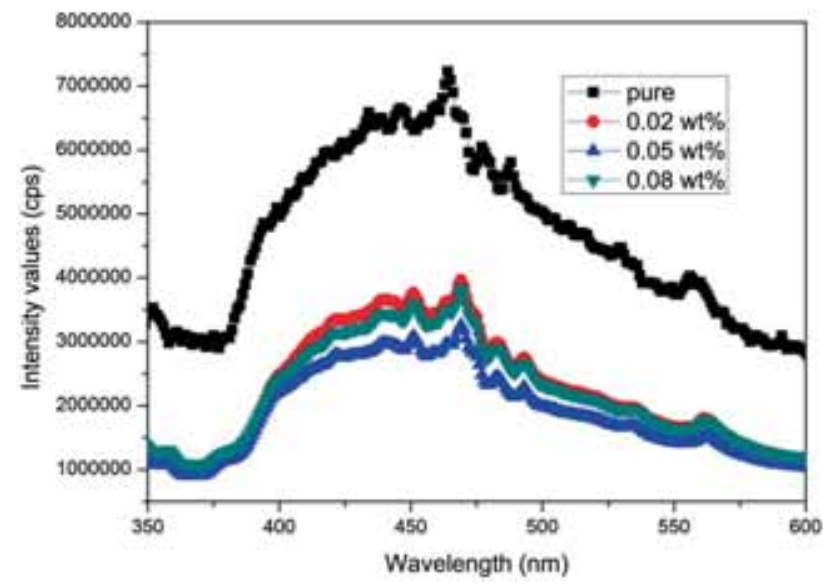

Figure 7. PL spectra of prepared samples.

incorporation up to $0.05 \mathrm{wt} \%$. And it possibly demonstrates the concentration quenching of PL emission. The incorporation of larger $\mathrm{Pr}^{3+}$ will produce charge imbalance and trapping centres within the system. These trapping centres could induce cross-relaxation and nonradiative transitions, resulting in quenching effect. But, while increasing the doping concentration, the possibility for the recombination increases and thus enhances the PL emission. The red shift of peaks observed in DL emission is a confirmation for the incorporation of $\mathrm{Pr}$ in $\mathrm{ZnO}$ lattice. And it can be connected with the reduction in band gap and particle size of the samples happened with doping.

\section{Photocatalytic analysis}

Photocatalytic activity was carried out using LZC-4XLuzchem photoreactor. The photoreactor is equipped with six sets of lamps and eight side lamps. It consists of two complete sets of UVA $(350 \mathrm{~nm})$ and UVC (254 nm) lamps as well as a safety goggles, a recessed magnetic stirrer and a power meter. The activity of the prepared photocatalysts was done by taking an aqueous solution of methylene blue (MB) (99.99\% purity, Merck product) of $10^{-5}$ molar concentration as a model pollutant. catalyst of $0.1 \mathrm{~g}$ was used to degrade $50 \mathrm{ml}$ of MB solution. The MB solution with photocatalyst is continuously stirred for $30 \mathrm{~min}$ without irradiation to reach adsorption-desorption equilibrium between MB molecules and catalyst surface. The distance between the light source of the reactor and $\mathrm{MB}$ solution was kept constant at $30 \mathrm{~cm}$ in all the cases. The reaction mixture was then irradiated under constant stirring and an amount of $5 \mathrm{ml}$ was withdrawn at regular intervals and centrifuged to separate the catalyst. UV absorption spectra of these solution samples were taken to analyse the degradation, based on the decrease in intensity of the characteristic peak of MB at $664 \mathrm{~nm}$, at different time intervals using UV-Vis spectrophotometer.

Figure 8 represents the time-dependent UV-Vis absorption spectra of the photocatalytic degradation of methylene blue in the presence of pure and Pr-doped $\mathrm{ZnO}$ catalysts. The pure $\mathrm{ZnO}$ is also an acceptable photocatalyst since it degrades the $94.23 \%$ of dye within $100 \mathrm{~min}$. But Pr incorporation enhanced the activity of $\mathrm{ZnO}$. The time taken for the degradation of $\mathrm{MB}$ dye is reduced to $30 \mathrm{~min}$ with the optimum incorporation of Pr. The experiment was repeated and error calculation was done to ensure the repeatability of the experiment. The absorption spectra with error bars confirms the repeatability. Figure 9 represents the degradation efficiency of $\mathrm{MB}$ with pure and $\mathrm{Pr}$-doped $\mathrm{ZnO}$ catalysts as a function of time. The efficiency of photocatalytic degradation is calculated using the formula, efficiency in percentage, $e=\left(\left(C_{0}-C_{\mathrm{t}}\right) / C_{0}\right) * 100$, where $C_{0}$ is the initial concentration and $C_{\mathrm{t}}$ the final concentration of MB. Photocatalytic efficiency of Pr-doped samples is very much higher than that of pristine. The maximum photocatalytic efficiency is shown by $0.05 \mathrm{wt} \%$ Pr-doped $\mathrm{ZnO}$ sample. The activity of the catalyst in the degradation of $\mathrm{MB}$ decreases, while the concentration of Pr exceeds the optimum value, but still better than pure $\mathrm{ZnO}$.

The reaction rate, $k$ of the degradation is found using Langmuir-Hinshelwood model. According to that model, a first-order kinetics can be expressed as

$$
\ln \left(C_{0} / C\right)=k t
$$

Using equation (4), the kinetic rate can be found from the slope of $\ln \left(C_{0} / C\right) v s$. time graph. Figure 10 represents the $\ln \left(C_{0} / C\right) v s$. time plot, which is used to find the kinetic rate of degradation reaction of MB with the prepared samples. Figure 11 is showing the kinetic rate of degradation reaction, while catalysts with different Pr content were used. The bar diagram indicates that the maximum kinetic rate is observed for $0.05 \mathrm{wt} \%$ Pr-doped sample, which facilitate the optimization of doping concentration. The kinetic rate of pure $\mathrm{ZnO}$ is $283.2 * 10^{-4}$ and for $0.05 \mathrm{wt} \%$ Pr-doped $\mathrm{ZnO}, 891.6 * 10^{-4}$. 

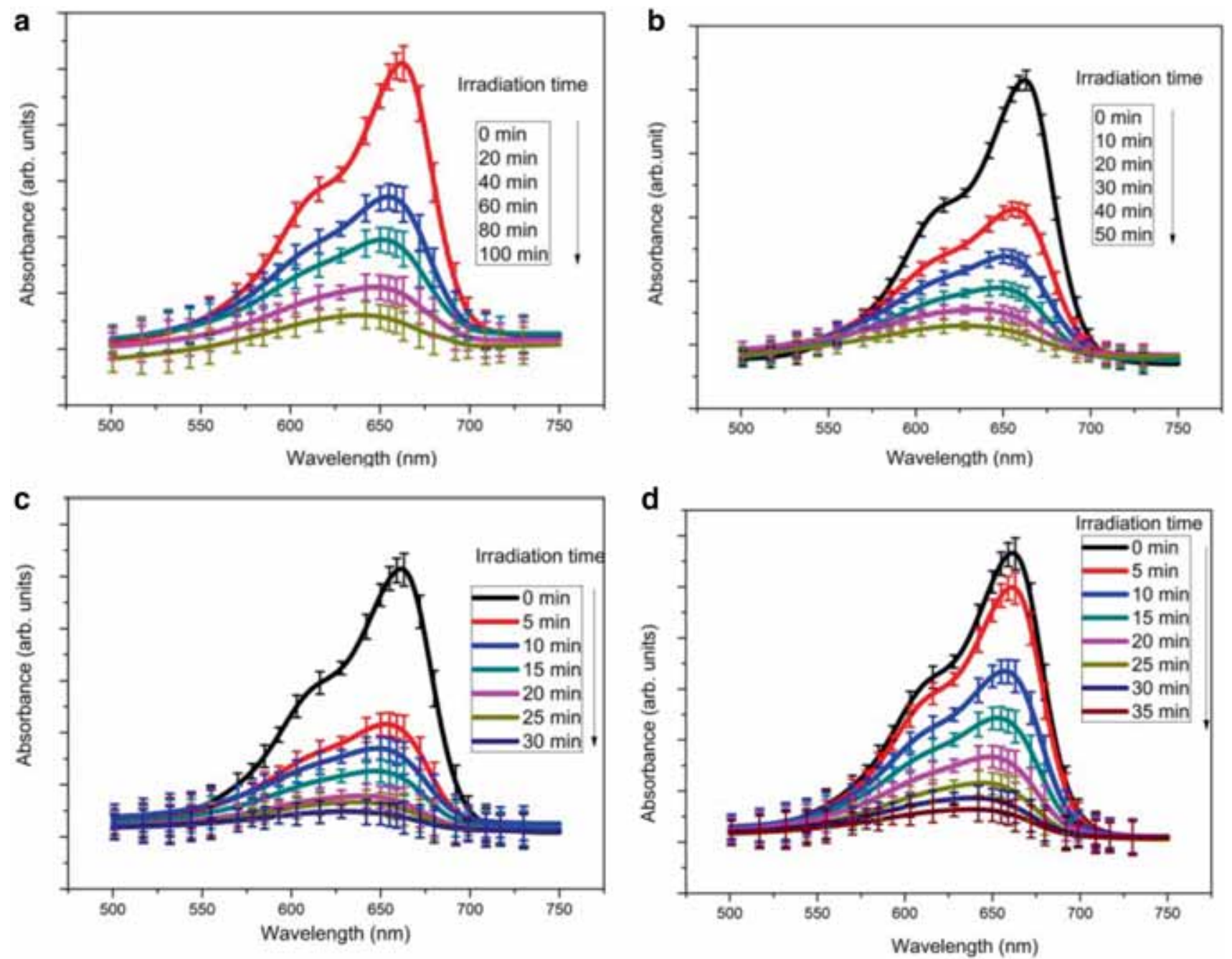

Figure 8. Time-dependent UV-Vis absorption spectra of the photocatalytic degradation of methylene blue in the presence of pure and Pr-doped ZnO samples. (a) Pure ( $\mathrm{Pr}=0)$, (b) 0.02 wt $\%$ Pr-doped $\mathrm{ZnO}$, (c) 0.05 wt $\%$ Pr-doped ZnO and (d) 0.08 wt $\%$ Pr-doped ZnO.

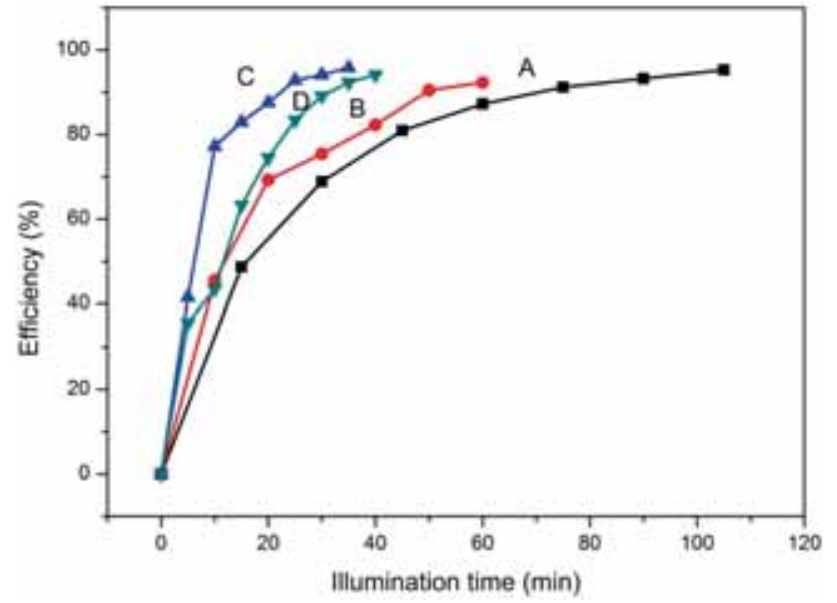

Figure 9. Degradation efficiency as a function of time curves of the methylene blue photodegradation using various concentrations of $\mathrm{Pr}$ in $\mathrm{ZnO}$ photocatalyst. A, pure $(\mathrm{Pr}=0)$; B, $0.02 \mathrm{wt} \%$ Pr-doped $\mathrm{ZnO}$; C, 0.05 wt\% Pr-doped ZnO; D, 0.08 wt\% Pr-doped ZnO.

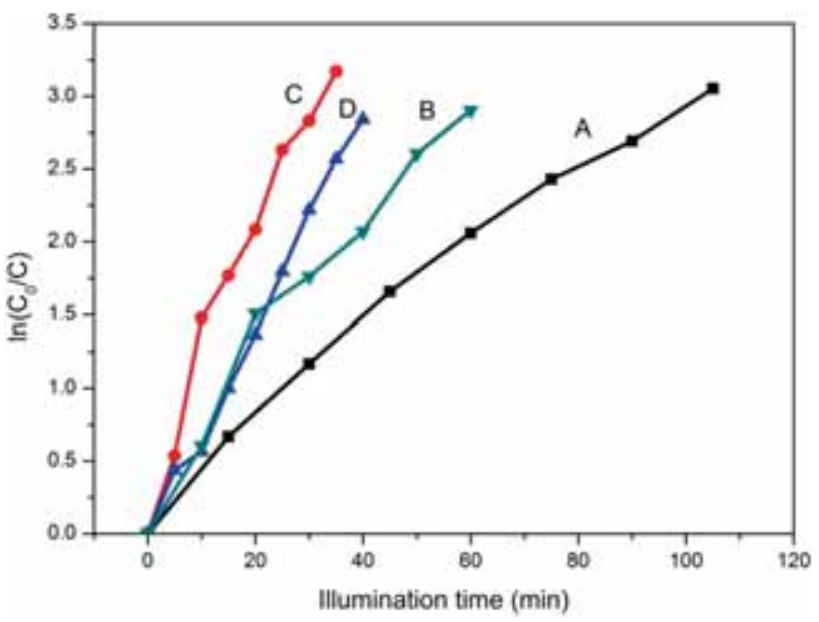

Figure 10. $\ln \left(C_{0} / C\right)$ vs. time graph of the prepared samples. A, pure $\mathrm{ZnO}$; B, 0.02 wt \% Pr-doped $\mathrm{ZnO}$; C, 0.05 wt\% Pr-doped $\mathrm{ZnO}$; and $\mathrm{D}, 0.08 \mathrm{wt} \%$ Pr-doped $\mathrm{ZnO}$. 


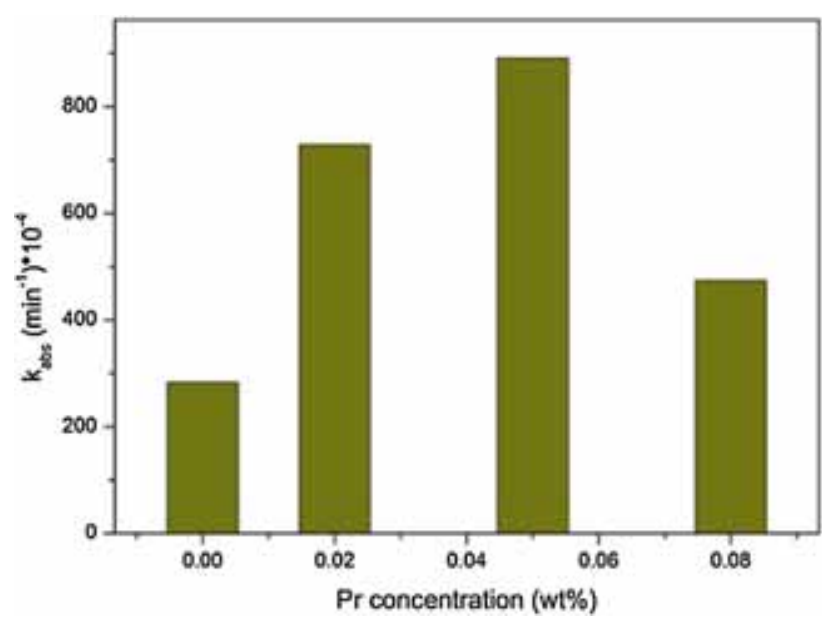

Figure 11. Degradation rate constant of prepared photocatalysts with different Pr content.

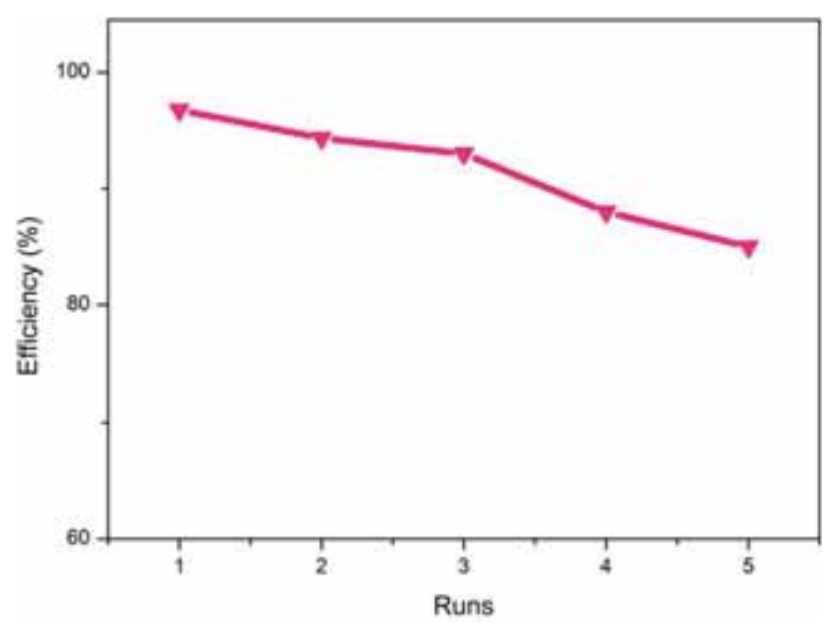

Figure 12. The stability of $0.05 \mathrm{wt} \%$ Pr-doped $\mathrm{ZnO}$ photocatalyst under UV irradiation. Irradiation time is fixed at $30 \mathrm{~min}$ for each run.

This result shows a three-fold enhancement of photocatalytic activity can be achieved with optimum Pr incorporation.

The practical applicability of the catalyst was checked with a stability test using $0.05 \mathrm{wt} \% \mathrm{Pr}$ - doped sample as the representative catalyst. The catalyst is separated at the end of each run. The collected catalyst is washed using distilled water and dried at room temperature. This dried and washed catalyst was used again for next run. The time of irradiation, the catalyst, the amount of catalyst, the quantities of MB solution as well as the initial concentration of MB was kept constant for each run during the whole stability test and the process is repeated for five more runs. The efficiency of the catalyst was calculated in each run. Figure 12 shows the reusability of $0.05 \mathrm{wt} \%$ Pr-doped $\mathrm{ZnO}$ catalysts for MB degradation. Even though, the degradation efficiency of the catalyst feebly decreases after each run, the catalyst kept good activity, even at sixth run. The catalyst succeeded in

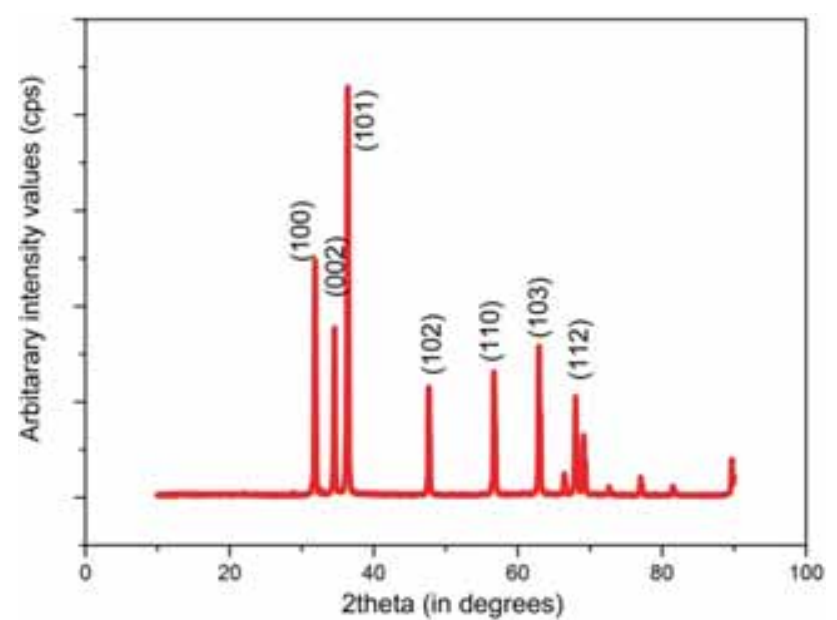

Figure 13. XRD of the used photocatalyst after use (Pr- $0.05 \mathrm{wt} \%$ doped $\mathrm{ZnO}$ ).

degrading $85 \%$ of $\mathrm{MB}$ within $30 \mathrm{~min}$, at the fifth run. The practical applicability of the samples was confirmed from this result. The chemical stability of catalysts was confirmed using XRD analysis. The used catalyst were well-washed with distilled water and dried at room temperature and taken for XRD analysis. Figure 13 represents the XRD spectrum of the used catalyst, which indicates that there is no chemical phase change occurs to the catalyst during the process of photocatalysis.

\subsection{Photocatalytic activity mechanism in $\mathrm{Pr}$-doped $\mathrm{ZnO}$ system}

As per the experimental analysis, it is seen that a three-fold enhancement in the photocatalytic activity has been achieved via Pr doping. A deep level analysis on the system is required to understand this enhancement. The UV light illumination makes electron-hole pair production in the system as per the equation below:

$$
\mathrm{ZnO}+h v \longrightarrow \mathrm{h}^{+}+\mathrm{e}^{-} .
$$

This electron gets interacted with the surface-adsorbed oxygen molecules and produce super oxide radical anions as shown in the equation below:

$$
\mathrm{e}^{-}+\mathrm{O}_{2} \longrightarrow{ }^{\circ} \mathrm{O}_{2}^{-} \text {. }
$$

The produced hole reacts with the surface hydroxyl groups and produce hydroxyl radicals as per the equation below:

$$
\mathrm{h}^{+}+\mathrm{OH} \longrightarrow{ }^{\circ} \mathrm{OH} \text {. }
$$

The holes produced via UV illumination is also able to dissociate water molecules in the aqueous solution of MB as per 
the chemical reaction shown below:

$$
\mathrm{h}^{+}+\mathrm{H}_{2} \mathrm{O} \longrightarrow \mathrm{H}^{+}+{ }^{\circ} \mathrm{OH}
$$

The produced oxygen and hydroxyl radicals are very powerful oxidants and they are able to degrade the organic pollutants into water and $\mathrm{CO}_{2}$. Here $\mathrm{ZnO}$ itself is a good photocatalyst and the efficiency of $\mathrm{ZnO}$ is improved via $\mathrm{Pr}$ doping. Pr doping has made a tailing effect in the band gap of $\mathrm{ZnO}$, enables high light absorption and enhanced the available number of charge carriers.

The incorporation of impurity ions within the $\mathrm{ZnO}$ matrix will introduce new defects within the system in addition to the intrinsic defects. The rare-earth (RE) elements have a dissimilar ionic radii as compared to the cation ( $\mathrm{Zn}$ ion) in the system. The incorporation of Pr ions wthin the system may cause the replacement of zinc cations and result in the production of defect like,

$$
\mathrm{Pr}_{\mathrm{Zn}}^{\circ} \longrightarrow+1 \text { net charge. }
$$

(Here we have used the Kroger Vink notation of defects.) The introduction of this defect results in the production of large number of electrons within the system to maintain the charge neutrality. And there is a chance of occupation of praseodymium ions within the interstitial positions of $\mathrm{ZnO}$ matrix. It may cause the defect like,

$$
\operatorname{Pr}_{i}^{\prime \prime \prime} \longrightarrow+3 \text { net charge. }
$$

This production of net positive charge is compensated by the production of more number of electrons to maintain the charge neutrality. The number of praseodymium ions that can accommodate in the $\mathrm{ZnO}$ matrix is very less. But the accommodated ions surely produce electron carriers in the system. But the band tailing happened in the band gap through defect levels will trap these electrons and thus inhibit the recombination. Thus, photoluminescence quenching happens in the system on Pr doping. But, the produced electrons are very effective to produce super oxide radical anions as per equation (6), which thereby increase the possibility of oxidation of pollutants effectively. This result in the enhancement of photocatalytic efficiency of Pr-doped $\mathrm{ZnO}$. Here, maximum photocatalytic efficiency is shown by $0.05 \mathrm{wt} \%$ Pr-doped $\mathrm{ZnO}$ sample. As the concentration of Pr increases, the number of charge carriers gets increased within the system, which in turn increases the probability for the recombination. Hence, at higher doping concentration, PL emission increases and photocatalytic activity decreases. After an optimum incorporation of impurity ion, the activity is seen decreased, but still better than the pure one. Particle size reduction as well as the band gap reduction happened on Pr doping has also contributed to the enhancement of photocatalytic efficiency of the system.

\section{Conclusion}

Praseodymium-doped $\mathrm{ZnO}$ nanoparticles within the solubility limit were successfully synthesized via modified solid-state reaction route. The structural, morphological and optical properties of prepared photocatalysts were investigated. The incorporation of $\mathrm{Pr}$ in $\mathrm{ZnO}$ was confirmed using EDAX analysis. Since, RE elements have larger ionic radii compared to zinc, the incorporation of trivalent Pr ions into $\mathrm{ZnO}$ host lattice produced a significant distortion in the $\mathrm{ZnO}$ crystal lattice. The crystallite size is calculated using Scherrer formula and is found to be decreased with increase in doping. The intensity of Raman peaks has decreased with the reduction in particle size. The optimum incorporation of $\mathrm{Pr}$ in $\mathrm{ZnO}$ has changed the irregular-shaped larger crystals to nanocrystals of relatively regular shape. As doping concentration increases, the Urbach energy increase within the system and result in the reduction of band gap energy. The band tailing process happened through Pr doping has positively contributed to photocatalytic activity. Fluorescent quenching occurs in the Pr-doped $\mathrm{ZnO}$ samples, because of the production of trapping centres. Defect production happens in the $\mathrm{ZnO}$ system on doping is analysed in detail. The defect production is correlated with PL and photocatalysis successfully. A three-fold enhancement of photocatalytic activity has achieved by the optimum incorporation of $\mathrm{Pr}$ in $\mathrm{ZnO}$. This work opens a path for the large-scale production of Pr-doped $\mathrm{ZnO}$ photcatalyst with good activity. The practical applicability of catalyst was confirmed from the stability test.

\section{Acknowledgements}

UGC-SAP support for this investigation (No. F.7-180/2007 (BSR)) is gratefully acknowledged. PPP thanks DST-SERBGovt. of India (SB/EMEQ-002/2013), DST-FIST-Govt. of India, Department of Physics, University of Calicut, for financial assistance and equipment facility.

\section{References}

[1] Ishizum Y and Kanemitsu I 2005 Appl. Phys. Lett. 86253106

[2] Jiang F, Peng Z, Zang Y and Fu X 2013 J. Adv. Ceram. 2201

[3] Chen G Y, Liu H C, Somesfalean G, Sheng Y Q, Liang H J, Zhang Z G et al 2008 Appl. Phys. Lett. 92113114

[4] Rodnyi P A and Khodyuk I V 2011 Opt. Spectrosc. 111776

[5] Yi J, Huang L, Wang H, Yu H and Peng F 2015 J. Hazard. Mater. 284207

[6] Vignesh K, Suganthi A, Rajarajan M and Sara S A 2012 Powder Technol. 224331

[7] Flores N M, Pal U, Galeazzi R and Sandoval A 2004 RSC Adv. 4 41099

[8] De Lourdes Ruiz Peralta M, Pal U and Sanchez Zeferino R 2012 Appl. Mater. Interf. 44807 
[9] Susarrey-Arce A, Herrera-Zaldivar M, de la Cruz W and Pal U 2009 J. Nanores. 5177

[10] Mohandes F and Salavati-Niasari M 2013 Mater. Res. Bull. 48 3773

[11] Schneider J, Matsuoka M, Takeuchi M, Zhang J, Horiuchi Y, Anpo M et al 2014 Chem. Rev. 114 9919

[12] Saleh R and Djaja N F 2014 Superlatt. Microstruct. 74217

[13] Li X and Ye J 2007 J. Phys. Chem. C 11113109

[14] Yayapao O, Thongtem T, Phuruangrat A and Thongtem S 2015 Mater. Sci. Semicond. Process. 39786

[15] Zhao Z, Song J-L, Zheng J-H and Lian J-S 2014 Trans. Nonferr. Met. Soc. China 241434

[16] Divya N K and Pradyumnan P P 2016 Mater. Sci. Semicond. Process. 41428
[17] Kim S Y, Lim T-H, Chang T-S and Shin C-H 2007 Catal. Lett. 117112

[18] Willander M, Nur O, Sadaf J R, Qadir M I, Zaman S, Zainelabdin A et al 2010 Mater. 32643

[19] Divya N K and Pradyumnan P P 2017 J. Mater. Sci.: Mater. Electron. 282147

[20] Choi H C, Jung Y M and Kim S B 2005 Vibr. Spectrosc. 37 33

[21] Ghosh M, Dilawar N, Bandyopadhay A K and Raychaudhari A K 2009 J. Appl. Phys. 106084306

[22] Divya N K and Pradyumnan P P 2017 Mater. Res. Exp. 4 015904

[23] John R and Rajakumari R 2012 Nano-Micro Lett. 465

[24] Klubnuan S, Suwanboon S and Amornpitoksuk P 2016 Opt. Mater. $\mathbf{5 3} 134$ 